\title{
LAGRANGIAN MAGNETO-HYDRODYNAMICS BASED ON CURVILINEAR FINITE ELEMENTS
}

\author{
JAN NIKL ${ }^{1}$, MILAN KUCHAŘ́íK ${ }^{2}$ AND STEFAN WEBER ${ }^{3}$ \\ ${ }^{1}$ ELI Beamlines Centre, Institute of Physics, Czech Academy of Sciences, Za Radnicí 835, Dolní \\ Břežany 25241, Czech Republic \\ Institute of Plasma Physics, Czech Academy of Sciences, Za Slovankou 1782/3, Prague 18200, Czech \\ Republic \\ Faculty of Nuclear Sciences and Physical Engineering, Czech Technical University in Prague, Břehová \\ 7, Prague 11519, Czech Republic \\ E-mail: jan.nikl@eli-beams.eu \\ ${ }^{2}$ Faculty of Nuclear Sciences and Physical Engineering, Czech Technical University in Prague, \\ Břehová 7, Prague 11519, Czech Republic \\ E-mail: milan.kucharik@fjfi.cvut.cz \\ ${ }^{3}$ ELI Beamlines Centre, Institute of Physics, Czech Academy of Sciences, Za Radnicí 835, Dolní \\ Břežany 25241, Czech Republic \\ E-mail: stefan.weber@eli-beams.eu
}

Key words: magneto-hydrodynamics, Lagrangian hydrodynamics, finite element method, isoparametric elements, magnetic diffusion

\begin{abstract}
The magneto-hydrodynamic model is widely used for description of magnetized fluids in plasma dynamics, microfluidics, astrophysics and many other applications. In terms of modelling, the Lagrangian formulation is favourable for the rapid expansion during laser-target interaction for example. This is the case for inertial fusion and laboratory astrophysics applications, which are our primary interest. However, the proposed numerical method remains general and can be applied elsewhere. The conservation properties and divergence-free magnetic field are crucial aspects, which are not satisfied by the traditional numerical schemes. Here, the Lagrangian hydrodynamics using curvilinear finite elements is extended to the resistive magneto-hydrodynamics. An energy-conserving numerical scheme is formulated maintaining divergence-free magnetic field. The mixed finite element formulation provides theoretically arbitrary order of the spatial convergence and application on unstructured Lagrangian grids in multiple dimensions. An example of a physically relevant numerical simulation is presented.
\end{abstract}

\section{INTRODUCTION}

The problem of modelling magnetized fluids arises in many disciplines like plasma dynamics, microfluidics, astrophysics. Our interest lies mainly in the former, where the continuum plasma dynamics within the laser-target interaction encompasses the pre-pulse effects of ultra-intense laser pulses [1] or non-local radiation and electron heat transport $[2,3]$. However, the proposed numerical model can be applied to a wide variety of problems. In general, the non-relativistic fluid exposed to an external or spontaneous 
magnetic field is governed by the equations of classical magneto-hydrodynamics, which are reviewed in section 2. The numerical solution of the coupled non-linear system can be divided into two main approaches, Eulerian and Lagrangian. The Eulerian methods rely on a fixed computational discretization in the laboratory frame, whereas the Lagrangian methods follow motion of the fluid. Despite being formally equivalent, the latter approach becomes advantageous in the cases of large expansion with a free boundary, such as the expansion of the ablated matter into vacuum under irradiation by a laser.

The proposed numerical scheme is based on the finite element method (FEM), which provides flexibility in the choice of the polynomial orders of the discretization and topology of the computational mesh. The numerical method of magneto-hydrodynamics (MHD) extends the high-order curvilinear finite element method [4, 5], where the isoparametric finite elements perfectly suit the Lagrangian description, as further commented in section 3. A numerical solution of the MHD equations using high-order finite elements has been proposed before [6]. However, energy conservation was not considered. This work continues along this line, applying the recent formalism of [7], in order to formulate a conserving and consistent multi-dimensional high-order curvilinear finite element method for MHD. Flexibility and scalability of the numerical implementation are provided by the MFEM library $[8,9]$. In this work, we present only preliminary results to demonstrate the prominent features of the model.

\section{LAGRANGIAN MAGNETO-HYDRODYNAMICS}

The classical resistive magneto-hydrodynamics provides a description for collisionally dominant plasmas in magnetic fields, which are modelled as a magnetized quasi-neutral one-temperature fluid, provided the

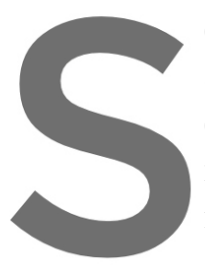
electron-ion relaxation pro and spatial scales. The energy $\varepsilon=\varepsilon(t, \vec{x})$ can be $\mathrm{d}$ is considered in this work nearly isotropic. Moreover, the apply the non-relativistic formulation. The equatio
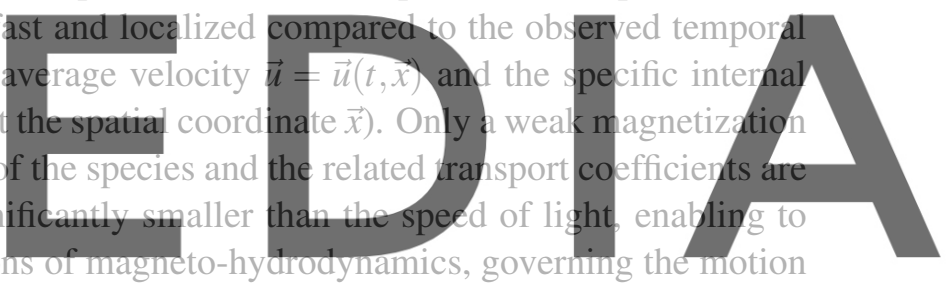

of the fluid and the magnetic field $\vec{B}=\vec{B}(t, \vec{x})$, are then taking the following form in the laboratory frame:

Register for free at https//www.scipedia.com to download the version without the watermark

$$
\begin{aligned}
\frac{\partial \rho}{\partial t}+\nabla \cdot(\rho \vec{u}) & =0, \\
\frac{\partial \rho \vec{u}}{\partial t}+\nabla \cdot\left(\rho \vec{u} \otimes \vec{u}-\overline{\bar{\sigma}}-\overline{\bar{\sigma}}_{B}\right) & =0, \\
\frac{\partial \vec{B}}{\partial t}+\nabla \cdot(\vec{u} \otimes \vec{B}-\vec{B} \otimes \vec{u}) & =-\nabla \times \vec{E}^{\prime}, \\
\frac{\partial}{\partial t}\left(\rho \varepsilon+\frac{1}{2} \rho \vec{u}^{2}\right)+\nabla \cdot\left(\left(\rho \varepsilon+\frac{1}{2} \rho \vec{u}^{2}\right) \vec{u}-\overline{\bar{\sigma}} \vec{u}\right) & =\vec{j} \cdot \vec{E}^{\prime},
\end{aligned}
$$

where $\overline{\bar{\sigma}}$ is the material stress tensor and $\overline{\bar{\sigma}}_{B}$ is the magnetic part of the Maxwell stress tensor $\left(\overline{\bar{\sigma}}_{B}=\right.$ $1 / \mu_{0}\left(\vec{B} \otimes \vec{B}-\frac{1}{2} \vec{B}^{2} \overline{\bar{I}}\right)$ with $\overline{\bar{I}}$ being the unit tensor). The vector field $\vec{j}=\vec{j}(t, \vec{x})$ represents the solenoidal currents following the electrostatic Ampère's law $\vec{j}=\mu_{0}^{-1} \nabla \times \vec{B}$, where $\mu_{0}$ is the permeability of vacuum (magnetization of the neutral background is not considered). Finally, the fluid-frame electric field is denoted as $\vec{E}^{\prime}$. The system of equations is closed by the equation of state, prescribing the value of the stress tensor $\sigma$ as a function of the state variables, and by Ohm's law $\vec{E}^{\prime}=\eta \vec{j}$, where $\eta$ is the resistivity of the plasma. 
The system of equations (1-4) together with the closure relations presents a closed system, which can be solved in the Eulerian coordinates directly. However, the Lagrangian description, where the computational mesh follows the motion of the plasma, is preferred for the applications of interest as mentioned in the introduction (section 1). The proper transformation of the coordinates to the fluid frame gives rise to the Lagrangian formulation [7]:

$$
\begin{aligned}
\frac{\mathrm{d} \rho}{\mathrm{d} t} & =-\rho \nabla \cdot \vec{u}, \\
\rho \frac{\mathrm{d} \vec{u}}{\mathrm{~d} t} & =\nabla \cdot\left(\overline{\bar{\sigma}}+\overline{\bar{\sigma}}_{B}\right), \\
\frac{\mathrm{d} \vec{B}}{\mathrm{~d} t} & =-\nabla \times \vec{E}^{\prime}, \\
\rho \frac{\mathrm{d} \varepsilon}{\mathrm{d} t} & =\overline{\bar{\sigma}}: \nabla \vec{u}+\vec{j} \cdot \vec{E}^{\prime}, \\
\rho \frac{\mathrm{d} \varepsilon_{B}}{\mathrm{~d} t} & =\overline{\bar{\sigma}}_{B}: \nabla \vec{u}-\frac{1}{\mu_{0}} \vec{B} \cdot \nabla \times \vec{E}^{\prime},
\end{aligned}
$$

\section{where $\mathrm{d} / \mathrm{d} t$ is the material derivative.}

The additional equation (9) governs the evolution of the specific magnetic energy, which is defined in the laboratory frame as $\varepsilon_{B}=\vec{B}^{2} /\left(2 \mu_{0} \rho\right)$. As it can be observed, the total energy is conserved, since it holds on the Lipschitz domain $\Omega$ with isolating boundary conditions:
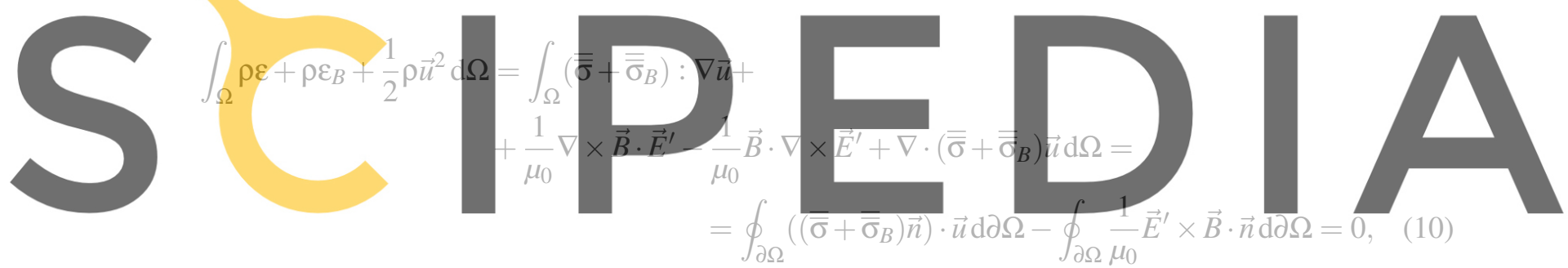

Register for free at https//www.scipedia.com to download the version without the watermark where the condition on zero normal forces and normal Poynting vector were used in the last equality ( $n$ is the outer normal at $\partial \Omega$ ). Following this formulation, an energy-conserving numerical scheme can be constructed in a tractable way within the Lagrangian framework.

\section{CURVILINEAR FINITE ELEMENT DISCRETIZATION}

The numerical scheme of the presented magneto-hydrodynamic model is based on the finite element method (FEM), where a mixed formulation is constructed. In the following, the computational mesh can be unstructured and topologically non-uniform in general. However, we limit ourselves to discussion and examples only on structured meshes consisting of quadrilateral elements for simplicity.

The proposed mixed formulation of the system (5-9) involves four different functional spaces on $\Omega$. The thermodynamic space $\mathcal{T}$ is used for the primary variables $\varepsilon$ and $\varepsilon_{B}$ and the kinematic space $\mathcal{K}$ for the velocity $\vec{u}$. The magnetic field $\vec{B}$ is an element of the corresponding space $\mathcal{M}$ and the electric field $\vec{E}^{\prime}$ is taken from the electric space $\mathcal{E}$.

The choices of the spaces are summarized in Table 1 for different dimensions. They build on the curvilinear high-order Lagrangian hydrodynamic scheme proposed earlier [4, 5], where the thermodynamic 


\begin{tabular}{l|ccc} 
space name & $\mathbf{1 D}(\| / \perp)$ & $\mathbf{2 D}(\| / \perp)$ & 3D \\
\hline thermodynamic $(\mathcal{T})$ & & $L_{2}$ & \\
kinematic $(\mathcal{K})$ & $\left(H^{1}\right)^{1}$ & $\left(H^{1}\right)^{2}$ & $\left(H^{1}\right)^{3}$ \\
magnetic field $(\mathcal{M})$ & $L_{2} /\left(L_{2}\right)^{2}$ & $H_{\text {div }} / L_{2}$ & $H_{\text {div }}$ \\
electric field $(\mathcal{E})$ & $-/\left(H^{1}\right)^{2}$ & $H_{\text {curl }} / H^{1}$ & $H_{\text {curl }}$
\end{tabular}

Table 1: Summary of the functional spaces for different dimensions.

potentials are discontinuous and the kinematic quantities are continuous in all directions. For the newly appearing spaces $\mathcal{M}$ and $\mathcal{E}$, it must be distinguished between the coplanar and transversal components in $1 \mathrm{D}$ and 2D, which are denoted by the symbols $\|$ and $\perp$, respectively. The choice of the magnetic space $\mathcal{M}$ follows the consideration about satisfaction of magnetic Gauss's law $\nabla \cdot \vec{B}=0$. This divergence-free magnetic field is maintained exactly by the definition of $\mathcal{M}$ and $\mathcal{E}$, as de Rham complex $H_{d i v} \stackrel{\nabla_{\perp} \times}{\longleftarrow} H^{1}$ holds in $2 \mathrm{D}\left(\nabla_{\perp} \times\right.$ is curl of the out-of-plane component) and $H_{d i v} \stackrel{\nabla \times}{\longleftarrow} H_{\text {curl }}$ in 3D [10]. Faraday's law (7) is then exact for the approximations of the functions and keeps the divergence of $\vec{B}$ intact.

The weak formulation of the system (5-9) is obtained by substitution of the primary quantities $\vec{u}, \vec{B}, \varepsilon$, $\varepsilon_{B}$ and $\vec{E}^{\prime}$ by their approximative counterparts from the respective functional spaces. For better readability, the identical symbols are used for the approximations henceforth, as the meaning is clear from the context. The weak formulation can then be written as:
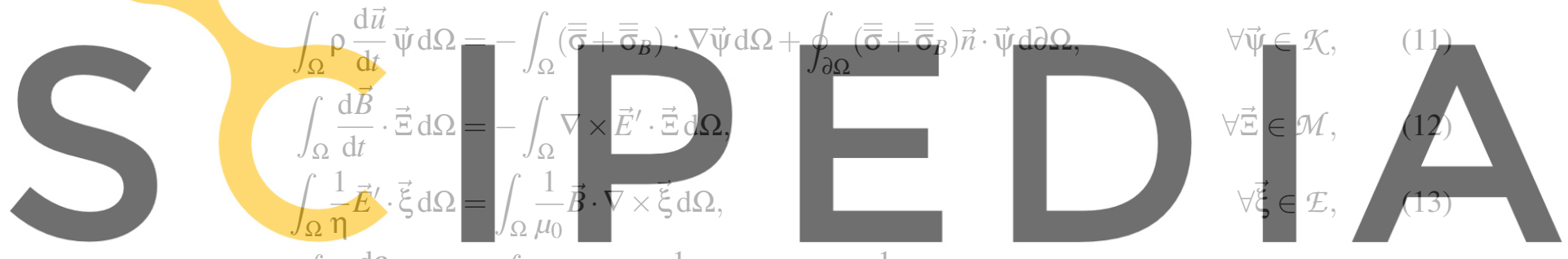

Register for free atehttps//Ww

$$
\begin{array}{cl}
-\oint_{\partial \Omega} \frac{1}{\mu_{0}} \mathrm{~T} \varphi\left(\vec{E}^{\prime} \times \mathrm{T} \vec{B}\right) \cdot \vec{n} \mathrm{~d} \partial \Omega, & \forall \varphi \in \mathcal{T}, \\
\int_{\Omega} \rho \frac{\mathrm{d} \varepsilon_{B}}{\mathrm{~d} t} \varphi \mathrm{d} V=\int_{\Omega} \overline{\bar{\sigma}}_{B}: \nabla \vec{u} \varphi-\frac{1}{\mu_{0}} \vec{B} \cdot \nabla \times \vec{E}^{\prime} \varphi \mathrm{d} V, & \forall \varphi \in \mathcal{T},
\end{array}
$$

where the operator T represents the trace of the functions on $\partial \Omega$. The equation of mass conservation is not present here, since the curvilinear high-order Lagrangian hydrodynamics undergoes the geometrical conservation law, i.e., the density is inverse proportional to the local Jacobian [5]. This shows the advantage of the isoparametric finite elements, where the strong conservation of mass is satisfied point-wise.

The essential points of construction of the numerical scheme are the weak forms and consequent symmetries between the equations, which give rise to conservation properties for arbitrary orders of the finite elements. The symmetry between the momentum equation (11) and the energy equation (14) leads to conservation of the kinetic energy, as shown already in [5]. Here, it is extended for the magnetic stress and its action in (15). Furthermore, the weak formulation of the Joule heating term $1 / \mu_{0} \nabla \times \vec{B} \cdot \vec{E}^{\prime}$ in (14) introduces a symmetry between the energy equations, providing conservation of the discrete energy in turn. Finally, a symmetry between the equation of the magnetic field and the energy equations exists, 
which leads to conservation of the magnetic energy on the discrete level. However, complete proofs are beyond the scope of this brief paper.

The formulation of the discrete scheme is not presented here for brevity, but it is done in the classical way on the conforming subspaces of $\mathcal{T}, \mathcal{K}, \mathcal{M}$ and $\mathcal{E}$ on a tessellation of $\Omega$. The hydrodynamic part of the scheme (including the magnetic energy equation) is solved in an explicit manner, where RK2Avg scheme is applied as in the original method $[4,5]$. It should be stressed that the discontinuous nature of the space $\mathcal{T}$ implies that the energy equations can be solved in each element separately, where computational costs related to the additional equation are marginal. The magnetodynamic part of the scheme, composed of Faraday's law (12) and Ohm's law (13), is solved semi-implicitly by the CrankNicolson scheme to be able to handle strong diffusion of the magnetic field.

\section{NUMERICAL RESULTS}

This section presents an example of numerical results obtained with the scheme described in section 3 . The studied problem is known as MHD blast, originally appearing in the context of extragalactic jets [11]. A significant portion of energy is placed at the center of the simulation domain initially and a strong magnetic field in the horizontal direction is imposed. A magnetosonic blast wave forms and propagates through space. Due to the only minuscule resistivity the magnetic field lines freeze in the expanding matter and are convected along with it.

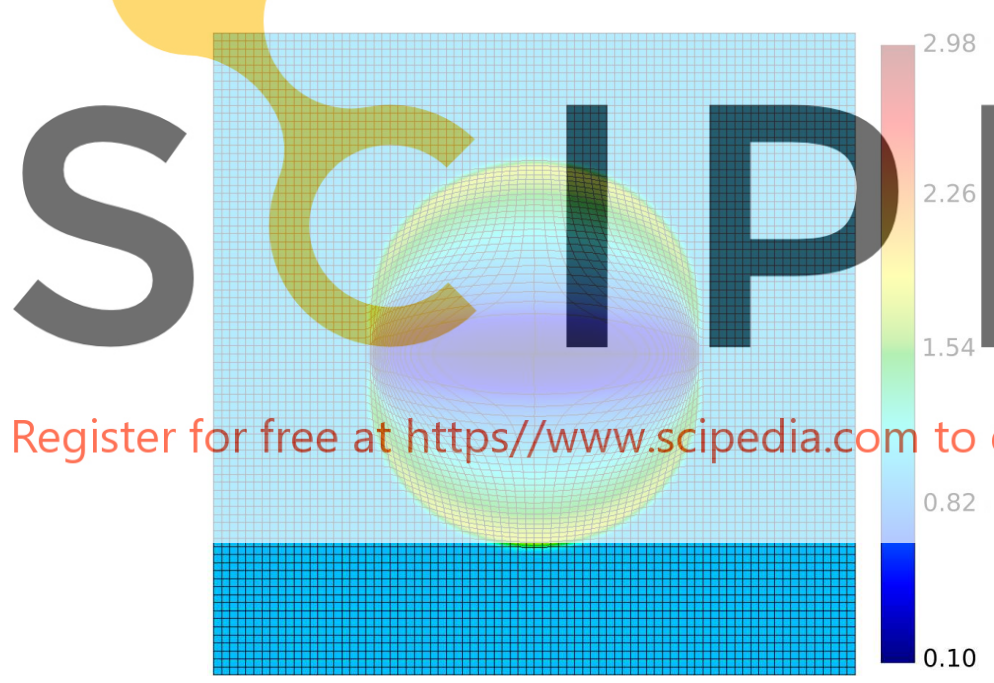

(a) Magnitude of the magnetic field (norm. to init. value)
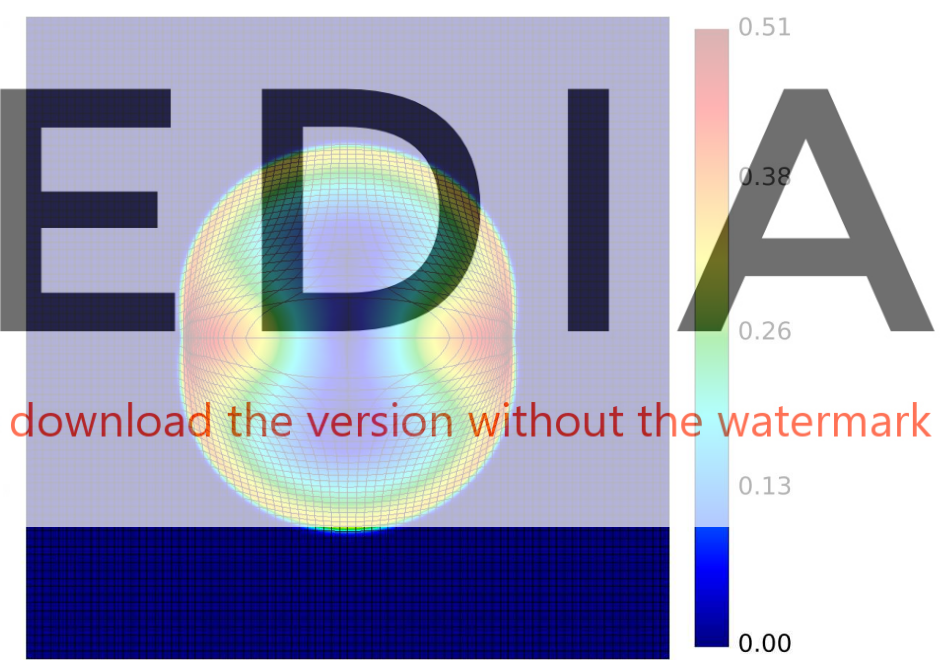

(b) Magnitude of velocity $(\mathrm{cm} / \mathrm{s})$

Figure 1: Spatial profiles of the quantities in the MHD blast problem at time $t=0.5 \mathrm{~s}$ for the quadratic $\mathcal{T}$ and $\mathcal{M}$ elements and cubic $\mathcal{K}$ and $\mathcal{E}$ elements. The resolution is $80 \times 80$ elements in each dimension. See the accompanying text for further details.

The parameters are set to $\rho \equiv 1 \mathrm{~g} / \mathrm{cm}^{3}$, the initial internal energy is $1 / 4$ erg deposited at the center of the domain spanning from $-1 \mathrm{~cm}$ to $+1 \mathrm{~cm}$. The ideal gas equation of state is used with the Poisson constant $\gamma=1.4$, atom mass number $A=1$, and constant ionization $Z=0$. Magnetic field in the horizontal direction has value corresponding to the magnetic pressure proportional to the thermal pressure. Specifically, $B^{2} /\left(2 \mu_{0}\right)=4 p_{\text {avg }}$, where $p_{\text {avg }}$ is the thermal pressure corresponding to the initial central energy equally 
distributed over the whole domain. The diffusion time of the magnetic field is set to $10^{3} \mathrm{~s}$.

The numerical results in Figure 1 show the prominent features of the scheme. The simulation is performed with the quadratic elements for $\mathcal{T}$ and $\mathcal{M}$ spaces and cubic elements for $\mathcal{K}$ and $\mathcal{E}$, where the resolution of the uniform (initially) quadrilateral computational mesh is only $80 \times 80$ elements. The higher order elements exhibit almost no mesh imprinting, despite the very low resolution. Also a very strong deformation of the elements is visible in the central region and compression near the fronts of the jet. This again diminishes the mesh imprinting, where the curvature of the isoparametric elements follows the shape of the blast wave.

\section{CONCLUSIONS}

For high-order numerical solution of the MHD equations in multiple dimensions, satisfying the divergencefree constraint and conservation of all velocity moments is non-trivial. Therefore, a multi-dimensional conserving Lagrangian magneto-hydrodynamic scheme based on high-order curvilinear finite elements is proposed. The divergence-free structure of the magnetic field is preserved from the definition as well as conservation of the velocity moments for arbitrary polynomial orders of the finite elements. An example of the numerical results for the MHD blast problem is presented to show the benefits of the approach. Further analysis of the numerical properties of the scheme and verification on different physically relevant problems remains a topic of future work. While the application for multi-physics modelling of laser-target interaction in the context of pre-pulse effects and non-local transport [1,2] can benefit from the new numerical scheme definitely, the method is very general and can be applied in other areas of

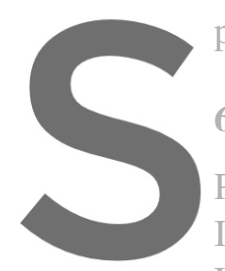
physics.

6 ACKNOWLEDGHMENTS

Portions of this research were carried out at ELI Institute of Physics of the Acad
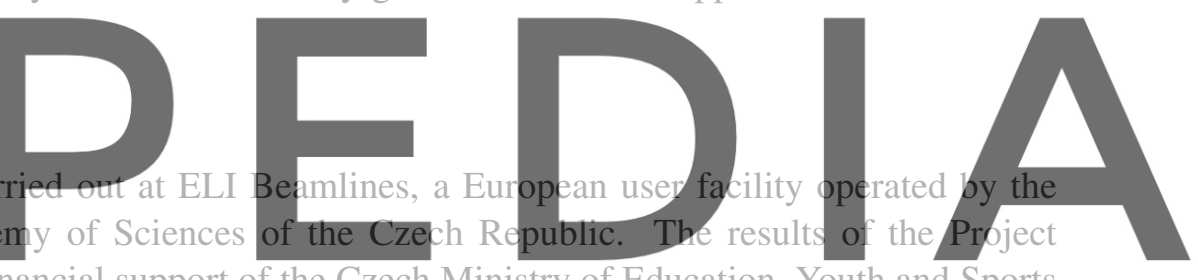

LQ1606 were obtained with the financial support of the Czech Ministry of Education, Youth and Sports

Register fas part of tafgeted support from the National Programme of Sustainability II. Supported by CAAS project versity grant SGS19/191/OHK4/3T/14 and Czech Science Foundation project 19-24619S. The computations were performed using computational resources funded from the CAAS project. This work has received funding from the Eurofusion Enabling Research Project No. ENR-IFE19.CEA-01.

Stefan Weber was supported by the project Advanced research using high intensity laser produced photons and particles (ADONIS) (CZ.02.1.01/0.0/0.0/16_019/0000789) and by the project High Field Initiative (HiFI) (CZ.02.1.01/0.0/0.0/15_003/0000449), both from European Regional Development Fund.

\section{REFERENCES}

[1] Holec, M., Nikl, J., Vranic, M., and Weber, S. The effect of pre-plasma formation under nonlocal transport conditions for ultra-relativistic laser-plasma interaction. Plasma Physics and Controlled Fusion (2018) 60(4):044019.

[2] Nikl, J., Holec, M., Zeman, M., Kuchařík, M., Limpouch, J., and Weber, S. Macroscopic laserplasma interaction under strong non-local transport conditions for coupled matter and radiation. Matter and Radiation at Extremes (2018) 3:110-126. 
[3] Holec, M., Nikl, J., and Weber, S. Nonlocal transport hydrodynamic model for laser heated plasmas. Physics of Plasmas (2018) 25(3):032704.

[4] Nikl, J., Kuchařík, M., Holec, M., and Weber, S. Curvilinear high-order Lagrangian hydrodynamic code for the laser-target interaction. Europhysics Conference Abstracts - 45th EPS Conference on Plasma Physics. Ed. by Coda, S., Berndt, J., Lapenta, G., Mantsinen, M., Michaut, C., and Weber, S. Vol. 42A. European Physical Society (2018), P1.2019.

[5] Dobrev, V. A., Kolev, Tz. V., and Rieben, R. N. High-Order Curvilinear Finite Element Methods for Lagrangian Hydrodynamics. SIAM Journal on Scientific Computing (2012) 34(5):B606B641.

[6] Rieben, R. N., White, D. A., Wallin, B. K., and Solberg, J. M. An arbitrary Lagrangian-Eulerian discretization of MHD on 3D unstructured grids. Journal of Computational Physics (2007) 226(1):534570.

[7] Wu, F., Ramis, R., and Li, Z. A conservative MHD scheme on unstructured Lagrangian grids for Z-pinch hydrodynamic simulations. Journal of Computational Physics (2018) 357:206-229.

[8] MFEM: Modular Finite Element Methods [Software]. https://mfem.org.

[9] Anderson, R., Andrej, J., Barker, A., Bramwell, J., Camier, J.-S., Cerveny, J., Dobrev, V., Dudouit, Y., Fisher, A., Kolev, Tz., Pazner, W., Stowell, M., Tomov, V., Akkerman, I., Dahm, J., Medina, D., and Zampini, S. MFEM: A modular finite element methods library. Computers \& Mathematics with Applications (2021) 81:42-74.

[10] Arnold, D. N., Falk, R. S., and Winther, R. Differential Complexes and Stability of Finite Element Methods I. The de Rham Complex. Compatible Spatial Discretizations. Ed. by Arnold, D. N.,

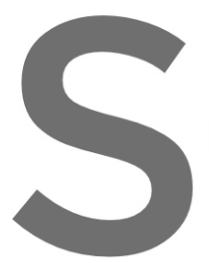
Bochev, P. B., Leh New York (2006) Kössl, D., Mülle tized jets, I : The ir 229(2):378-396
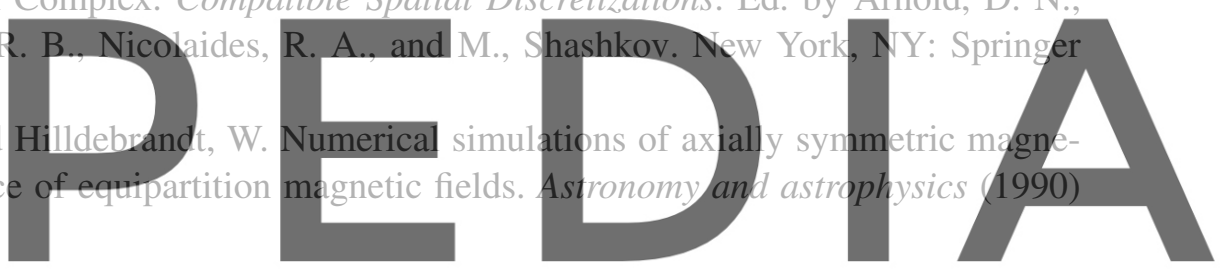

Register for free at https//www.scipedia.com to download the version without the watermark 